\title{
Réforme de l'Ordre des avocats en Colombie: entre économie de la concurrence et économie de la qualité*
}

\author{
Reforming the Bar in Colombia: between the economy of \\ Competition and Economics of Quality \\ Reforma del Colegio de Abogados en Colombia: entre la \\ economía de la Competencia y Economía de la Calidad \\ Recibido: 10 de mayo de 2010 - Revisado: 01 de junio de 2010 - Aceptado: 18 de julio de 2010
}

Edwin Tachlian**

\section{Résumé}

A l'heure de la réforme de la justice en Colombie, cet article vise à explorer la pertinence et l'efficacité de deux conceptions opposées de la réglementation de la profession d'avocat: l'autorégulation d'un ordre professionnel autonome et la libéralisation, c'est-à-dire l'absence de règles spécifiques liées à la profession libérale. Pour cela seront présentés divers modèles d'ordres d'avocat puis la position de la Commission Européenne sur la question. Enfin nous verrons en quoi cette position pourrait en réalité s'opposer à la garantie de qualité du service.

Mots Clés

Concurrence, ordre professionnel, avocats, reforme de la justice en Colombie.

\section{Resumen}

Al momento de la reforma de la justicia en Colombia, este artículo propone analizar la pertinencia y la eficacia de dos concepciones rivales de la regulación de las profesiones libérales y particularmente de la profesión de abogado: la autorregulación a través de una orden profesional independiente, y la liberalización, es decir, la ausencia de normas específicas para la profesión. Para eso se presentarán distintos modelos de asociaciones de abogados antes de analizar la posición de la Comisión Europea sobre el tema. Por último, veremos cómo esta posición puede efectivamente oponerse a la calidad de servicio garantizada.

Palabras clave

Competencia, ordenes profesionales, abogados, reforma de la justicia en Colombia.

\footnotetext{
Abstract

At the time of the reform of justice in Colombia, this article aims to explore the relevance and effectiveness of two competing conceptions of the regulation of professions, particularly the legal profession: self-regulation through a professional order, and liberalization, ie the absence of specific rules related to the profession. For that will be presented various models of lawyers' associations before to analyze the European Commission's position on that issue. Finally we will see how this position could effectively oppose the guaranteed quality of service. Keywords Competition, professional, lawyers, justice reform in Colombia.
}

* Artículo resultado de investigación. Línea de análisis crítico de las instituciones. Grupo Crear. Universidad Sergio Arboleda.

** Enseignant Chercheur, Groupe CREAR, Ecole de Droit, Université Sergio Arboleda. Research Professor, Crear Group School of Law, Sergio Arboleda University. Docente Investigador, Grupo Crear Escuela de Derecho, Universidad Sergio Arboleda. Correo electrónico:

edwin.tachlian@usa.edu.co 
L'investiture de Juan Manuel Santos à la présidence de la Colombie et l'arrivée de son administration ont été accompagnées d'un long projet de réforme judiciaire. Parmi les diverses propositions du gouvernement, le projet de loi de réforme de la justice prévoit dans son l'article 24 une modification de la constitution en son article 257 A. "- Est de compétence du Collège National des avocats, l'élaboration $d u$ registre national des avocats ; le pouvoir d'enquête et de sanction en cas de manquements à la discipline des avocats dans l'exercice de leur profession, conformément à la loi.

La profession d'avocat, jusqu'à présent contrôlée par la chambre administrative du Conseil Supérieur de la Magistrature par le biais de son Registre et Contrôle des Avocats et Auxiliaires de justice, se retrouve donc sur le chemin de l'autorégulation par ses propres membres. Le futur Collège sera responsable de l'exercice du contrôle disciplinaire de la fonction juridique au niveau national, tandis qu'au même moment, de l'autre coté de l'atlantique, la Commission Européenne s'est prononcée dès 2004 en faveur de la libéralisation des professions libérales, qui serait accompagnée de la disparition effective des différents ordres nationaux d'avocats.

En effet, la Commission Européenne semble assimiler les ordres professionnels à des cartels institutionnalisés en mesure d'imposer des barrières à l'entrée sur le marché des services juridiques (par exemple des conditions minimales pour exercer la profession) et de faciliter les accords sur les prix entre ses membres. Si tel était le cas, les ordres professionnels, comme celui des avocats, s'opposeraient aux principes de la concurrence dans le marché commun résultant des ex articles 81 et 82 du traité de Rome. D'un autre point de vue cependant, ne pourrait-on pas considérer que les membres d'une profession sont les mieux à même de réguler leur activité et d'assurer ainsi la qualité du service offert ? De ce phénomène se pose naturellement la question de la place des professions libérales dans un marché concurrentiel, entre économie de marché et économie de qualité.

Cet article vise donc à explorer la pertinence et l'efficacité de ces deux conceptions opposées de la réglementation des professions libérales, et particulièrement celle de la profession d'avocat. Pour cela seront présentés divers modèles d'ordres d'avocat puis la position de la Commission Européenne sur la question. Enfin nous verrons en quoi cette position pourrait en réalité s'opposer à la garantie de qualité du service.

\section{Modèles d'Ordres nationaux des Avocats : Organisation et Concurrence}

\section{1) Colombie}

La profession est actuellement organisée autour du Conseil supérieur de la magistrature, un organisme chargé de l'administration et de la délivrance de la carte professionnelle d'avocat.

La Loi 1123 de 2007 établit le Code disciplinaire de l'avocat. Le paragraphe 15 de son article 28 prévoit l'obligation pour tous les avocats d'être inscrits et de maintenir à jour une domiciliation professionnelle au Registre National des Avocats. L'inscription doit être faite et déposée auprès de la salle administrative des conseils de section de la magistrature.

\section{Loi 1123 DE 2007}

ARTICLE 28. Obligations professionnelles des AVOCATS. Les devoirs de l'avocat:

«15. Avoir une adresse professionnelle connue, enregistrée et mise à jour au Registre national des avocats à l'attention des questions qui lui sont confiées, doit également signaler immédiatement tout changement de celle-ci aux autorités avec lesquelles se fait toute gestion professionnelle. » 
En outre s'applique le décret 196 de 1971, modifié par la loi 583 de $2000^{1}$, qui donne le statut de la pratique de la profession:

« La loi est la fonction sociale de la collaboration avec les autorités dans la conservation et l'amélioration des institutions juridiques nationales, et la mise en œuvre d'une administration correcte et complète de la justice. La mission principale est de défendre la justice défendre les droits de la société et les individus. Sa mission consiste également à conseiller, de parrainer et d'aider les personnes dans la gestion et le développement de leurs relations juridiques. Est avocat qui a obtenu le grade de conformité avec les exigences universitaires et juridiques. »

Il est pour la pratique nécessaire d'être enregistré comme avocat, sans préjudice des exceptions énoncées dans le présent décret.

L'inscription estobligatoirepourl'obtention $d u$ titre correspondant, légalement reconnu par l'État. Nul ne peut exercer la profession d'avocat ou de la publicité en tant que tel sans être inscrit et ayant une immatriculation valide.

Ne peut être enregistré comme avocat et si l'est doit être exclu:

a. Qui est en interdiction judiciaire, et

b. Le responsable de délit condamné à une peine d'emprisonnement ou de bannissement, commis après l'entrée en vigueur du présent décret, si de par les conditions et circonstances de l'acte, les motivations et les déterminants de la personnalité de l'agent, le tribunal compétent a considéré comme indigne de la pratique du droit.

A l'exception de la condamnation avec sursis ou pardon judiciaire.

\section{b) France}

C'est au cours du XIIIe siècle qu'est né en France la profession d'avocat. L'avocat, au sens étymologique (Ad vocatus»: celui qui s'exprime à la place.), aide les autres à la justice. Conformément à la réglementation régissant la profession (la première datant de 1274), l'avocat est tenu de prêter serment et d'être inscrit sur une liste officielle .

La principale activité des avocats est la défense. L'adhésion au barreau est la première étape et représente un pas vers une carrière dans la magistrature ou l'administration. Mais le développement des frais de vente (depuis le XVIe siècle) fait qu'il est plus difficile d'accèder à la fonction publique. Le barreau a ainsi tendance à affirmer son identité et son autonomie vis à vis du pouvoir judiciaire.

Au XVIIIe siècle, le barreau affirme son indépendance par rapport au gouvernement, agissant librement dans les grands débats qui agitent la France de l'avant Révolution. Celle-ci est bien accueillie par de nombreux avocats, mais son travail demeure inséparable de l'ancienne administration de la justice sous 1'Ancien Régime et disparaît avec celle-ci en 1790.

Aujourd'hui, la profession d'avocat est une profession réglementée. En ce sens, le titre d'avocat est un titre protégé.

Utiliser le titre d'avocat doit en effet coïncider avec l'exercice de la profession. Ne peuvent faire usage du titre que ceux qui ont obtenu le diplôme et inscrits à l'ordre. La loi du 31 Décembre 1990 relative à la protection des professions réglementées parle en conséquence du «droit de périmètre». Nul ne peut, s'il n'est pas une partie des professions qui sont autorisées, plaider, représenter, conseiller ou préparer des documents juridiques. Cette protection est avant tout une garantie pour le défendeur d'être sûr qu'il est lié à une personne qui a reçu une formation spé- 
ciale, qui a une compétence spécifique, qui est soumis aux règles d'éthique, dont le respect est garantis par une autorité supérieure, le Conseil de l'Ordre. (Barreau de Paris).

L'usurpation du titre d'avocat est par conséquent pénalement punissable d'un an d'emprisonnement et d'une amende de 15.000 euros. De même, toute personne qui n'est pas avocat, mais effectue une ou plusieurs activités du champs réservé de la profession peut être punie de six mois d'emprisonnement et de 4500 euros d' amende (Barreau de Paris).

\section{c) États-Unis}

Aux États-Unis, les avocats sont admis à exercer dans un Etat particulier. Comme critères d'admission, les avocats doivent passer un examen, généralement contrôlé par un comité d'examinateurs de l'État. L'American Bar Association (ABA), fondée le 21 août 1878, est une association bénévole d'avocats et d'étudiants en droit, qui n'est pas spécifique à une juridiction d'État. L'activité principale de l'ABA est l'établissement de normes universitaires aux facultés de droit, et la formulation du modèle de codes d'éthique lié à la profession juridique. L'ABA est régie par la Présidence des Etats Unis, la Chambre des délégués, siégeant en tant que principal organe de l'Organisation pour l'adoption de nouvelles politiques et recommandations dans le cadre de la position officielle de l'association et le Conseil des gouverneurs. (American Bar Association, 2010).

Un rôle important de l'ABA est sa création et l'entretien d'un code de déontologie pour les avocats. Le Code modèle de la responsabilité professionnelle (1969) ou les nouvelles règles du modèle de conduite professionnelle de 1983 ont été adoptés dans 49 Etats et le District de Columbia. L'exception est la Californian Bar Association, cependant, certaines des sections du Code de déontologie pour la Californie proviennent des modèles de 1'ABA. (State Bar of California, 2010)

\section{La déréglementation de la profes- sion juridique dans l'Union européen- ne: les professions dans un marché parfaitement concurrentiel}

\author{
A) Concurrence dans l'Union européen- \\ ne: généralités
}

La création du Marché commun est l'un des objectifs majeur du traité de Rome de 1957. L'existence du marché commun est basée sur l'élimination des obstacles à la libre circulation des marchandises, des personnes, des capitaux et des services entre les États membres. Le Marché commun doit permettre aux entreprises de développer plus facilement leurs activités, générant ainsi des retombées économiques ainsi que des avantages aux consommateurs via une plus grande variété de produits à des prix plus compétitifs.

Pour atteindre ces objectifs d'ouverture du marché à la concurrence au sein de l'Europe, les institutions européennes sont fondées sur les articles 101 et 102 du Traité de Lisbonne (ex art. 81 et $82 \mathrm{TCE}$ ), qui ont introduit une interdiction sur les accords restreignant la concurrence et l'abus de position dominante. Les comportements qui faussent le mécanisme concurrentiel sont interdits, ainsi que ceux qui ont un effet sur le commerce interétatique et donc nuire à l'objectif de l'Union, et en particulier la formation de cartels en vertu de l'article $81 \mathrm{du}$ traité de 1957 qui fixe également la compétence de la Commission.

Article 101 du Traité de Lisbonne (ex art. 81 TCE):

1. Sont incompatibles avec le marché intérieur et interdits tous accords entre entreprises, décisions d'associations d'entreprises et toutes pratiques concertées, qui sont susceptibles d'affecter le commerce entre États membres et qui ont pour objet ou pour effet d'empêcher, de restreindre ou de fausser le jeu de la con- 
currence à l'intérieur du marché intérieur, et notamment ceux qui consistent à:

a) fixer de façon directe ou indirecte les prix d'achat ou de vente ou d'autres conditions de transaction,

b) limiter ou contrôler la production, les débouchés, le développement technique ou les investissements,

c) répartir les marchés ou les sources d'approvisionnement,

d) appliquer, à l'égard de partenaires commerciaux, des conditions inégales à des prestations équivalentes en leur infligeant de ce fait un désavantage dans la concurrence,

e) subordonner la conclusion de contrats à l'acceptation, par les partenaires, de prestations supplémentaires qui, par leur nature ou selon les usages commerciaux, n'ont pas de lien avec l'objet de ces contrats.

2. Les accords ou décisions interdits en vertu du présent article sont nuls de plein droit.

3. Toutefois, les dispositions du paragraphe 1 peuvent être déclarées inapplicables: à tout accord ou catégorie d'accords entre entreprises, à toute décision ou catégorie de décisions d'associations d'entreprises et

- à toute pratique concertée ou catégorie de pratiques concertées qui contribuent à améliorer la production ou la distribution des produits ou à promouvoir le progrès technique ou économique, tout en réservant aux utilisateurs une partie équitable du profit qui en résulte, et sans: a) imposer aux entreprises intéressées des restrictions qui ne sont pas indispensables pour atteindre ces objectifs,

b) donner à des entreprises la possibilité, pour une partie substantielle des produits en cause, d'éliminer la concurrence.

Alors la question se pose de savoir si ces articles 101 et 102 du traité de Rome s'appliquent aux avocats et procureurs des ordres nationaux.

B) L'interdiction des ententes: application aux ordres professionnels

Les règles sont appliquées lorsque la pratique anticoncurrentielle peut affecter le commerce entre États membres. Conformément à l'article $101 \mathrm{du}$ traité de Lisbonne du, sont prohibés les accords qui ont pour objet ou pour effet d'empêcher, de restreindre ou de fausser la concurrence dans le marché commun. Ces accords sont nuls et peuvent être sanctionnés par la Commission européenne d'une amende pouvant aller jusqu'à $10 \%$ du chiffre d'affaires mondial. Toutefois, l'application de cette règle aux ordres des avocats et autres professions suppose deux conditions: que l'avocat soit une entreprise, et que l'ordre d'un avocat soit une association d'entreprises dont le but est d'empêcher, de restreindre ou de fausser la concurrence (Cohen, 2010).

\section{C) L'avocat comme entreprise}

En Droit Communautaire, la notion de la société, dont la définition n'est pas dans le traité, est interprétée de manière extensive par la Commission et la Cour de justice de la Communauté, soit comme une entité exerçant une activité économique. Par activité économique, on entend offre de produits ou services dans un marché donné, sans avoir à déterminer si oui ou non l'activité est lucrative. La conséquence est que non seulement les entreprises mais aussi 
les individus peuvent être considérés comme des entreprises au sens des articles 81 et 82 . Dans le cas de Pavlov du 12 Septembre 2000, la Cour de Justice a jugé que des experts médicaux indépendants exerçent une activité économique et constituent donc une entreprise. En vérité, selon cette décision, les médecins «offre en sa qualité de commerçants indépendants, des services marchands, les services médicaux spécialisés ... qu'ils reçoivent des paiements pour les services de la part des patients et assument les risques financiers liés à l'exercice de leur activité. Dans la décision Pavlov, la Cour déclare: «Dans ces conditions, les experts indépendants qui participent à l'activité économique sont donc les entreprises .... sans que la complexité et la nature technique des services qu'ils fournissent et la circonstance que l'exercice de leur profession est réglementée ne puisse modifier cette conclusion. $»^{2}$.

En matière d'avocats, la même conclusion a été adoptée par le tribunal le 12 Janvier 1995 par le tribunal de première instance Européen dans sa décision Vitto.

\section{D) La position de la Commission euro- péenne}

Dans un rapport de 2004, la Commission Européenne reconnaît l'intérêt de limiter l'accès aux professions de bienfaisance afin de garantir la qualité du service. Cependant, basé sur une étude empirique de Cox $\boldsymbol{C}$. S. et Foster, qui a révélé que les restrictions quantitatives ont diminué la qualité du service, la Commission est manifestement opposée aux règles régissant les professions.

La Commission Européenne revendique ouvertement saposition favorableà la concurrence en accord avec la création et la protection du marché intérieur commun et libéralisé. En effet, la Commission, ayant adopté une ligne classique, considère les professions libérales comme des oligopoles institutionnalisées autour des ordres professionnels. La Commission se place donc en faveur d'un marché concurrentiel en favorisant la baisse des prix et la stimulation de l'innovation, l'autorégulation du marché limitant mécaniquement le nombre de professionnels en fonction du niveau de la demande. C'est la raison pour laquelle, dans le même rapport, la Commission propose de réduire les conditions d'accès aux professions et de réduire le champ réservé de la profession d'avocat.

\section{Concurrence de marché vs III. Eco- nomie de la qualité}

\section{A) L'Ordre national des avocats comme groupe d'intérêt pour l'Ecole du Public Choice}

Pour l'Ecole du Public Choice, les citoyens rationnels dans un système démocratique comprennent que la poursuite de leurs intérêts est plus efficace par l'action de groupe que par le vote individuel. L'objectif du groupe d'intérêt est donc l'obtention d'avantages de ses membres. Mais si l'objectif est de garantir l'intérêt commun des membres, la seule communauté d'intérêt ne garantit pas que les membres agissent de concert.

Or, chacun peut être tenté de profiter gratuitement des efforts du groupe (comportement de free-riding). Ce problème est particulièrement aigu dans un grand groupe. En revanche, les groupes d'intérêt sont généralement beaucoup plus faciles à construire que le nombre potentiel de membres est réduit. La formule la plus radicale pour surmonter le problème potentiel de free-riding est l'utilisation de la contrainte de l'État afin d'obliger une personne à rejoindre ce groupe (MacKaay, 2000). L'exercice d'une profession peut en effet être réservée par la loi aux membres du groupe, comme c'est le cas dans la plupart des pays pour les médecins, les notaires, les architectes et les finalement avocats.

Pour ces professionnels, l'adhésion obligatoire est justifiée par l'intérêt public, qui prend la forme de la protection du public contre l'incompétence. Obligatoire une fois établie, elle se révèle en fait être une restriction à l'entrée sur le marché, poussant à des prix plus 
élevés ceux qui se pratiqueraient sans intervention. (MacKaay, 2000).

Enfin, l'autorégulation, définie comme l'élaboration et le respect, par les acteurs euxmêmes, de règles qu'ils ont et dont ils assurent eux-mêmes l'application, (Du Marais, 2002) présente un caractère de club favorisant ses membres au détriment des acteurs potentiels. Cette caractéristique se trouve renforcée par le domaine réservé et par les conditions d'exercice de la profession. En pratique, l'autorégulation favorise donc la mise en place de barrières à l'entrée et de façon générale d'un comportement de cartel

b) L'Economie de la Qualité: une Vision Hétérodoxe du Marché

La Commission Européenne entend rapprocher le marché des services légaux d'un état de concurrence pure et parfaite (réunissant les conditions d'atomicité, d'homogénéité des produits, de transparence de l'information, de libre entrée et sortie sur le marché et de liberté de circulation des facteurs de production). Or, l'application d'une théorie orthodoxe du marché au cas d'espèce présuppose néanmoins que ledit marché soit lui aussi orthodoxe. Qu'en est il du marché des services juridiques?

La profession d'avocat se caractérise par une forte asymétrie de l'information entre le professionnel et son client (Chaserant et Harnay, 2010). En effet, comme beaucoup de professions intensives en connaissances, le client se trouve dans une position de confiance vis-à-vis de l'avocat. Le choix de l'avocat de la part du client se fera donc en premier lieu sur la spécialité de l'avocat (en effet, il est difficile de parler d'homogénéité des produits tant les missions et champs de connaissance diffèrent d'un avocat à un autre) mais encore sur sa réputation. Dans ce cadre, l'ordre professionnel assure, à travers son organe disciplinaire, la bonne réputation de la profession dans son ensemble en sanctionnant les écarts de conduite. En outre, ses règles permettent d'assurer un standard minimum de qualité pour l'ensemble des consommateurs, compensant ainsi l'asymétrie d'information entre le professionnel et son client, car qui d'autre que ses pairs peut assurer une évaluation fiable du service offert par un avocat?

\section{Le Cas Colombien: de la Régulation à l'Autorégulation}

En Colombie, la profession d'avocat, jusqu'à présent contrôlée par la chambre administrative du Conseil Supérieur de la Magistrature par le biais de son Registre et Contrôle des Avocats et Auxiliaires de justice, se retrouverait en théorie en état d'autorégulation. Le futur Collège National des avocats sera responsable de l'exercice du contrôle disciplinaire de la profession ainsi que de l'enregistrement obligatoire des avocats.

En effet, la proposition de loi de réforme à la justice de 2010 propose la création d'un article 257 A de la Constitution par lequel se créera le collège national des avocats. competent pour la gestion du registre national des avocats et pour enquêter et sanctionner les manquements à la discipline.

Cependant, s'agit-il réellement d'une transition vers l'autorégulation ? En effet, le futur article 257 A continu comme suit:

«La loi déterminera la composition et le fonctionnement du Collège National des Avocats.

(...)

CLAUSE DE TRANSITION. En attendant l'entrée en fonctionnement $d u$ Collège National des Avocats, la fonction disciplinaire doit être exercée par le Bureau du procureur général».

La composition et le fonctionnement du Collège National étant assuré par le pouvoir législatif, l'on peut se demander s'il s'agit 
bien là d'un ordre professionnel susceptible de s'autoréguler, ou plutôt d'une sorte de commission de régulation sectorielle. La loi éclaircira certainement ce point, en particulier les compétences en matière d'appel des décisions disciplinaires, de même que la compétence du Collège National en matière de rédaction de normes spécifiques à la profession sur le modèle des codes de déontologie et d'étique américains.

\section{Conclusion}

Les arguments visant à libéraliser les professions libérales, en particulier celle d'avocat, semblent à ce jour obsolètes, car ne prenant pas en compte les spécificités d'un marché qui, sans une régulation par ses propres membres, ne serait être bénéfique au consommateur. En effet, ce marché caractérisé par une forte asymétrie de l'information entre professionnel et client, ne peut garantir un standard minimum de qualité du service proposé par le professionnel que par l'évaluation de ses pairs. La Colombie semble se placer à contre courant de l'Union Européenne en la matière en proposant au sein de sa réforme de la justice, la création d'un Collège National d'Avocats en lieu et place de l'actuelle régulation à travers de la chambre administrative du Conseil supérieur de la magistrature. Cependant la question se pose de savoir s'il s'agit véritablement d'une autorégulation ou plutôt d'une co-régulation, définie comme " un lieu d'échange, de négociation entre les "parties prenantes" et les titulaires de la contrainte légitime et où se comparent les bonnes pratiques, afin de les ériger en recommandations. Ce lieu (pouvant) également servir d'instance de médiation » (Du Marais, 2004). Et finalement, où serait la réforme en passant d'un organe disciplinaire composé de magistrats (donc avocats de formation) à un organe où les représentants sont certes des avocats mais nommés par la loi.

\section{Notas}

${ }^{1}$ Décret 196 de 1971, modifié par la loi 583 de 2000.
${ }^{2}$ CJCE 12 Septembre 2000 Pavlov C $18 \mathrm{O} / 198$ et C 184 / 98 Points 74 et 76.

\section{References}

American Bar Association. Recuperé le 27 juin 2010, de: http://www.abanet.org/

Barreau de Paris http://www.avocatparis.org/

Benichou, M. (2008), rapport l'europe, les avocats \& la concurrence.

Chappuis, B. (2008) Droit de la concurrence et droit des avocats: la fin des tabous, in Mélanges en l'honneur de Pierre Tercier, p. 572.

Chaserant, C. et Harnay, S. (2010). Déréglementer la profession d'avocat en france ? Les contradictions des analyses économiques, Revue internationale de droit économique, 2010/2 (t.XXIV), De Boeck Université.

Cohen, C. (2010) Article n 40 : L'avocat est-il une entreprise et le barreau une association d'entreprises ? Ou de l'application a l'avocat et aux barreaux des articles 1 et $82 \mathrm{du}$ traite de l'union europeenne sur les regles de concurrence- a propos de l'interprofessionnalite et de l'arre. Recuperé le 27 juin 2010, de: http://www.barreauaixenprovence.avocat.fr/fr/profession/ deontologie/id-40-1-avocat-est-il-une-entreprise-et-le-barreau-une-association-dentreprises-ou-de-1-application-a-1-avocatet-aux-barreaux-des-articles-1-et-82-dutraite-de-1-union-europeenne-sur-lesregles-de-concurrence-a-propos-de-1-interprofessionnalite-et-de-1-arre (récupéré le $01 / 10 / 2010$ ).

Commission Européenne (2004) Rapport sur la concurrence dans le secteur des professions libérales. Communication de la Commission, [COM(2004) 83 final - Non publié au Journal officiel]. 
Cox C. et S. Foster, (1990) "The costs and benefits of occupational regulation", rapport du Bureau of Economics Staffà la commission fédérale du commerce, 1990, p. 26-27.

Du Marais, B. (2002). Analyses et propositions pour une régulation de l'Internet, Lex Electronica, vol. 7, $n^{\circ} 2$, Printemps.

Du Marais, B. (2004). Régulation de l'internet: des faux-semblants au retour à la réalité Revue française d'administration publique 2004/1 - N¹09 pp 83 - 91.

Mackaay, E. (2000). Analyse Economique du Droit, fondements.

Rapport «Monti» (2006). Sur Le suivi du rapport sur la concurrence dans le secteur des professions libérales (2006/2137(INI).

Rapport (2008) Les avocats entre ordre professionnel et ordre marchand, Concurrence par la qualité et socio-économie d'une réglementation professionnelle. Rapport Final.

The State Bar of California. Recuperé le 27 juin 2010, de: http://www.calbar.ca.gov/

\section{Décisions}

CJCE, 12 Septembre 2000 Pavlov C - 18O / 198 et C 184 / 98 Points 74 et 76.

Tribunal de Première Instance Européen, 12 janvier 1995 Vitto, T 102/92 Commission Rec. P.11-17 Article 50.

\section{Normes}

Traité de Lisbonne, Articles 101 et 102 du (ex art. 81 et 82 TCE).

Proyecto de Acto Legislativo - Reforma Judicial Constitucional, 25 aout 2010, Ministere de l'interieur et de la justice. (Colombie).
Loi 1123 De 2007 (Colombie).

Décret 196 de 1971, modifié par la loi 583 de 2000 (Colombie).

Code modèle de la responsabilité professionnelle (1969) (USA).

Modèle de Conduite Professionnelle de 1983 (USA).

Loi du 31 Décembre 1990 relative à la protection des professions réglementées (France). 
